Ronald Boellaard* Carsten Kobe

Josée M. Zijlstra

N. George Mikhaeel

Peter W.M. Johnson

Stefan Müller

Ulrich Dührsen

Otto S. Hoekstra

Sally Barrington

*VU University Medical Center

De Boelelaan 1117

1081 HV Amsterdam, The Netherlands

E-mail: r.boellaard@vumc.nl

Published online Apr. 6, 2018.

DOI: 10.2967/jnumed.118.211607

\section{Embrace Progress}

TO THE EDITOR: We would like to comment on the letters by Boellaard et al. in this issue of The Journal of Nuclear Medicine and Barrington et al. (1). The authors urge reporters not to use PET reconstruction algorithms, which exploit point-spread function (PSF) modeling or Bayesian penalized likelihood (BPL) techniques for response assessment in lymphoma. This call to ignore a more sensitive reconstruction technique, demonstrated to yield image data closer to phantom truth (2), and with the ability to detect smaller volume disease, is of concern. The Deauville criteria (DC) were designed to simplify and standardize how we interpret an ${ }^{18} \mathrm{~F}$-FDG PET/CT scan for the presence or absence of active lymphoma and to guide clinicians in the management and prognosis of their patients. The comment that there is "a shift toward more positive reads" is unfortunate as it references a letter by Barrington et al. that has only anecdotal evidence from 3 patients and is not a peer-reviewed paper or a largecohort series. We believe there is insufficient evidence to support the recommendation to not use these more sensitive PET/CT reconstruction methods.

Recent publications have emphasized that BPL PET reconstruction is particularly advantageous in patients with high body mass index (3), that is, in patients with the greatest background noise in whom the detection of small abnormalities is most problematic. This improvement in signal-tonoise ratio may improve inter- and intraobserver variation in assessing DC. With older reconstruction techniques, even among experts, interobserver agreement using the DC is only moderate (4), and it has been suggested that this may be because of difficulty comparing the signal in a lesion with noisy background signal in the liver or mediastinal blood (5). PSF and BPL still underestimate true activity in small foci, however, they are a step forward and nearer to phantom truth. This truth can make reporting more challenging, requiring careful consideration of the clinical significance of the detection of small-volume and subtle abnormalities.

The recent publication by Enilorac et al. (6) compared a PSF reconstruction with European Association of Nuclear Medicine (EANM) Research Ltd. (EARL)-compliant reconstruction in 126 diffuse large B-cell lymphoma patients. They concluded that neither DC score nor risk stratification of diffuse large B-cell lymphoma patients was significantly affected by the choice of PET reconstruction and that specifically the use of PSF is not an issue in routine clinical processes or in multicenter trials. In practice, there are probably few patients with discordant DC on ordered-subset expectation maximization versus more advanced reconstructions, with potential to alter management. It will be of im- mense value to study these patients, which may require collaboration between centers, so that going forward lessons can be learned. Nonetheless, it is also important to remember that the interpretation of interim scans and the decisions related to them are not binary. They should ideally be made in the clinical context, in relation to lymphoma type, stage, and risk factors, such as bulk or B symptoms and the intensity of treatment given before and after the PET scan (5).

Alongside the use of interim PET, it is important to remember that PET is used to more accurately stage lymphoma at presentation, with significant value in detecting extranodal disease, and this is likely to be further improved using more sensitive imaging techniques. The use of new reconstruction methods at baseline staging then effectively mandates its use for follow-up scans, as the detection of new or progressive disease remains important.

We would argue that early disease detection often leads to better treatment and clinical outcomes. We need to embrace technologic advances and innovation even if these lie outside our comfort zone. The current situation is very similar to any major advance in imaging, such as the transition from 2-dimensional to 3-dimensional PET reconstruction. However, this learning curve does not mean these advanced methods should be avoided; we would suggest that patients with malignancies should be staged and followed up as accurately as possible using the most sensitive technique available. This may require alteration to the DC as previously occurred with changes to the International Harmonization Project in 2014 after the increased use of more modern PET/CT scanners.

\section{REFERENCES}

1. Barrington SF, Sulkin T, Forbes A, Johnson PWM. All that glitters is not gold: new reconstruction methods using Deauville criteria for patient reporting. Eur $J$ Nucl Med Mol Imaging. 2018;45:316-317.

2. Teoh EJ, McGowan DR, Macpherson RE, Bradley KM, Gleeson FV. Phantom and clinical evaluation of the Bayesian penalized likelihood reconstruction algorithm Q.Clear on an LYSO PET/CT system. J Nucl Med. 2015;56:14471452 .

3. Chilcott AK, Bradley KM, McGowan DR. Effect of a Bayesian penalized likelihood pet reconstruction compared with ordered subset expectation maximization on clinical image quality over a wide range of patient weights. AJR. 2018;210:153157.

4. Ceriani L, Barrington S, Biggi A, et al. Training improves the interobserver agreement of the expert positron emission tomography review panel in primary mediastinal B-cell lymphoma: interim analysis in the ongoing International Extranodal Lymphoma Study Group-37 study. Hematol Oncol. 2017;35:548553.

5. Barrington SF, Kluge R. FDG PET for therapy monitoring in Hodgkin and nonHodgkin lymphomas. Eur J Nucl Med Mol Imaging. 2017;44:97-110.

6. Enilorac B, Lasnon C, Nganoa C, et al. Does PET reconstruction method affect Deauville score in lymphoma patients? J Nucl Med. 2018:59:1049-1055.

Kevin M. Bradley*
Daniel R. McGowan
Fergus V. Gleeson
Geoffrey B. Johnson
Jason R. Young
Craig S. Levin
Guido A. Davidzon
Andrei H. Iagaru
*Oxford University Hospitals NHS Foundation Trust
Oxford, OX3 7LE, U.K.
E-mail: lisa.meade@ouh.nhs.uk

Published online Apr. 26, 2018.

DOI: 10.2967/jnumed.118.212761 